\title{
LATTICES OF CONTINUOUS FUNCTIONS
}

\author{
IRVING KAPLANSKY
}

1. Introduction. Let $X$ be a compact (=bicompact) Hausdorff space and $C(X)$ the set of real continuous functions on $X$. By defining addition and multiplication pointwise, we convert $C(X)$ into a ring. With the norm $\|f\|=\sup |f(x)|, C(X)$ becomes a Banach space. Finally, we may introduce an ordering by defining $f \geqq g$ to mean $f(x) \geqq g(x)$ for all $x$; this makes $C(X)$ a lattice.

Gelfand and Kolmogoroff [6] $]^{1}$ showed that, as a ring alone, $C(X)$ characterizes $X$. More precisely, if $C(X)$ and $C(Y)$ are isomorphic rings, then $X$ and $Y$ are homeomorphic. Banach [3, p. 170] proved that $C(X)$ as a Banach space characterizes $X$, if $X$ is compact metric. Stone $[5$, p. 469] generalized this to any compact Hausdorff space, and Eilenberg [5] and Arens and Kelley [2] have since given other proofs. Finally, Stone [9] has shown that as a lattice-ordered group, $C(X)$ characterizes $X$. A negative result is that $C(X)$ as a topological linear space fails to characterize $X$ [3, p. 184].

In this paper we shall prove the following result: as a lattice alone $C(X)$ characterizes $X$. This theorem is shown in $\$ 5$ to subsume all the earlier results cited above. Moreover in this context we can replace the reals by an arbitrary chain, granted a suitable separation axiom. In $\S 4$ it is shown that the connectedness of $X$ is equivalent to the indecomposability of $C(X)$ as a lattice.

I am greatly indebted to Professor A. N. Milgram for suggestions which led to a substantial simplification of my proof of Theorem 1.

2. Main theorem. Let $R$ be a chain (simply ordered set). Until $\S 6$ it will be assumed that $R$ has neither a minimal nor maximal element. There is a natural way of topologizing $R[4$, p. 27] which can be described as follows: for any $\alpha \in R$ let $U(\alpha)$ be the set of all $\beta \in R$ with $\beta>\alpha, L(\alpha)$ the set of all $\beta$ with $\beta<\alpha$; then the $U$ 's and $L$ 's form a subbase of the open sets.

Lemma 1. If $\alpha, \beta \in R$ and $\alpha>\beta$, then there exist neighborhoods $M, N$ of $\alpha, \beta$ such that $\gamma>\delta$ for all $\gamma \in M, \delta \in N$.

Proof. If there exists $\xi$ with $\alpha>\xi>\beta$ we take $M=U(\xi), N=L(\xi)$. If not, we take $M=U(\beta), N=L(\alpha)$.

Received by the editors January 2, 1947.

${ }^{1}$ Numbers in brackets refer to the bibliography at the end of the paper. 
Let $X$ be a topological space and $C=C(X)$ the set of all continuous functions from $X$ to $R$. We order $C$ by defining $f \geqq g$ to mean $f(x) \geqq g(x)$ for all $x \in X$. Then $C$ becomes a lattice, and in fact a distributive lattice.

Definition. $X$ is $R$-separated if for any $x, y \in X(x \neq y)$ and $\alpha, \beta \in R$ there exists a continuous function $f$ with $f(x)=\alpha, f(y)=\beta$. $X$ is $R$-normal, if for any disjoint closed sets $F, G \subseteq X$ and any $\alpha, \beta \in R$ there exists a continuous function $f$ equal to $\alpha$ on $F$ and $\beta$ on $G$.

Any $R$-separated space is necessarily a Hausdorff space. If $R$ is the real number system, it is known that conversely any compact Hausdorff space is $R$-normal, and this is likewise true if $R$ is the set of reals with $\pm \infty$ adjoined (the latter is the same as a bounded closed interval on the real line). If $R$ is disconnected, it is clear that $X$ must be totally disconnected if it is to be $R$-separated, and conversely a totally disconnected space is $R$-separated for every $R$. In the extreme case where $R$ consists of two elements, $C(X)$ is a Boolean algebra in its natural ordering, and the results in this paper are subsumed in Stone's theory of Boolean spaces and rings [7].

For any $R$ we have the following result, which can be proved in exactly the same way as the lemma on p. 487 of [1].

Lemma 2. Suppose $R$ has neither a maximal nor minimal element. Then if $X$ is compact and $R$-separated, it is $R$-normal.

By a prime ideal $P$ in $C[4$, p. 78] we mean the set of antecedents of 0 in some lattice homomorphism of $C$ onto the two-element lattice $(0, I)$. A prime ideal $P$ is a sublattice containing with any element all smaller ones, and its complement $C-P$ has the dual property.

We can construct prime ideals in $C$ as follows. Let $Z$ be the lower half of a Dedekind cut in $R$, and for a fixed point $x \in X$, let $P$ consist of all $f$ with $f(x) \in Z$. Such prime ideals can be characterized as follows: $f \in P$ and $g(x) \leqq f(x)$ imply $g \in P$. We shall see below ( $\$ 3)$ that these do not in general exhaust all prime ideals. However, we can prove a certain weakened version of this property.

Definition. A prime ideal $P$ in $C$ is associated with a point $x \in X$ if $f \in P$ and $g(x)<f(x)$ imply $g \in P$.

LEMMA 3. If $X$ is compact, then any prime ideal $P$ in $C$ is associated with some point. If $X$ is $R$-separated, the point is unique.

Proof. Suppose $P$ is associated with no point of $X$. Then for every $x \in X$ we have functions $f, g$ with $g(x)<f(x)$ and $f \in P, g \in Q=C-P$. By Lemma 1 the inequality $g<f$ extends to a neighborhood of $x$. A 
finite number of these neighborhoods cover $X$. Let $f_{1}, \cdots, f_{n}$ and $g_{1}, \cdots, g_{n}$ be the corresponding functions, and define $h=f_{1} \cup \cdots \cup f_{n}$, $k=g_{1} \cap \cdots \cap g_{n}$. Then $h>k$, but $h \in P, k \in Q$, a contradiction.

Suppose now that $X$ is $R$-separated and that $P$ is associated with both $x$ and $x^{\prime}$. Let $m, n$ be any functions in $P, Q$ respectively. There exists a function $r$ with $r(x)<m(x), r\left(x^{\prime}\right)>n\left(x^{\prime}\right)$. But these inequalities require $r$ to be in both $P$ and $Q$.

LEMMA 4. If two prime ideals in $C$ are associated with the same point of $X$, then their intersection contains a prime ideal.

Proof. Suppose $P$ and $P^{\prime}$ are both associated with $x$. Let $f \in P$, $g \in P^{\prime}$ and take any $\alpha \in R$ smaller than both $f(x)$ and $g(x)$. Let $P^{\prime \prime}$ be the set of all $h$ with $h(x) \leqq \alpha$; then $P^{\prime \prime} \subset P \cap P^{\prime}$.

Lemma 5. Suppose $X$ is compact and $R$-separated. Let $P, P^{\prime}, P^{\prime \prime}$ be prime ideals in $C$ with $P^{\prime \prime} \subset P \cap P^{\prime}$. Then $P$ and $P^{\prime}$ are associated with the same point.

Proof. Suppose on the contrary that $P$ and $P^{\prime}$ are associated with distinct points $x$ and $y$. Let $P^{\prime \prime}$ be associated with $z$, where $z$ is for definiteness different from $x$. Choose any $f$ in $P^{\prime \prime}, g$ in $C-P$, and then $h$ with $h(z)<f(z), h(x)>g(x)$. Then $h$ is in $P^{\prime \prime}$ but not in $P$, a contradiction.

For use in $\$ 4$ we insert at this point the following result.

Lemma 6. Suppose $X$ is compact. If $P$ is a prime ideal associated with $x, f \in P$, and $g \leqq f$ on an open and closed set $U$ containing $x$, then $g \in P$.

Proof. Suppose $g \in Q=C-P$. We note that $P$ cannot be associated with a point $y$ in the complement $V$ of $U$, for we can construct a function $m$ with $m(x)<f(x), m(y)>g(y)$ and obtain a contradiction. Hence at every $y \in V$ we have functions $h, k$ with $h>k$ in a neighborhood of $y$ and $h \in P, k \in Q$. A finite number of neighborhoods cover the compact space $V$; if $h_{1}, \cdots, h_{n}, k_{1}, \cdots, k_{n}$ are the corresponding functions we have $f \cup h_{1} \cup \cdots \cup h_{n}$ in $P$ but exceeding $g \cap k_{1} \cap \cdots \cap k_{n}$ in $Q$.

LemMa 7. Suppose $X$ is compact and $R$-separated. Let $f_{0}$ be a fixed function in $C$, and for any subset $S$ of $X$ define $A(S)$ to be the intersection of all prime ideals containing $f_{0}$ which are associated with points of $S$. Then a point $x$ is in the closure of $S$ if and only if $A(S)$ is contained in a prime ideal associated with $x$.

Proof. Suppose $x$ is in the closure of $S$. Choose $\alpha>f_{0}(x)$ and let $P$ 
consist of all $f$ with $f(x) \leqq \alpha$. Now $g \in A(S)$ implies $g \leqq f_{0}$ at all points of $S$ and hence $g(x) \leqq f_{0}(x)$. It follows that $A(S) \subset P$. (For later use we remark that this half of the proof did not use $R$-separation.)

Suppose $x$ is not in the closure of $S$. Then $A(S)$ cannot be contained in any prime ideal $P$ associated with $x$. For let $f$ be any function in $C-P$ and let $\alpha \in R$ be chosen with $f_{0}>\alpha$ on $S$. By Lemma 2, a continuous function $g$ exists which equals $\alpha$ on $S$ and exceeds $f$ at $x$. Then $g$ is in $A(S)$ but not in $P$, a contradiction.

We can now prove our principal theorem.

THEOREM 1. Let $R$ be a chain with neither a minimal nor maximal element, and let it be endowed with its order topology. Let $X$ be a compact $R$-separated space, and $C$ the set of continuous functions from $X$ to $R$. Then as a lattice, $C$ characterizes $X$.

Proof. We say that two prime ideals in $C$ are equivalent if their intersection contains a third prime ideal. Lemmas 3,4 , and 5 show that there is a one-one correspondence between points of $X$ and classes of equivalent prime ideals. Lemma 7 shows that the topology of $X$ can be expressed in terms of inclusion relations among the prime ideals, and this completes the proof.

3. An example. We shall give an example to show that not all prime ideals in $C$ are simply based on a point in $X$ and a Dedekind cut in $R$.

Let $R$ be the reals, $X$ the unit interval $[0,1]$. Let $A$ be the set of functions $f$ for which $f(x) \leqq-x$ in a neighborhood of 0 (the neighborhood depending on $f$ ). $A$ is an ideal in $C$, that is, a sublattice containing with any element all smaller ones. Similarly let $B$ be the set of all $g$ with $g(x) \geqq x$ in a neighborhood of $0 ; B$ is a dual ideal disjoint from $A$. By [8, Theorem 6] we can expand $A, B$ to a prime ideal $P$ and its complementary dual prime ideal $Q$. All functions $f$ with $f(0)<0$ go into $P$, all with $f(0)>0$ go into $Q$, but those vanishing at 0 are split between $P$ and $Q$.

Examples of this type can be constructed provided neither $X$ nor $R$ is discrete. They apparently require the axiom of choice.

4. Connectedness and direct products. If the space $X$ splits into two open and closed sets $X_{1}$ and $X_{2}$, it is evident that the lattice $C=C(X)$ is the direct product $[4, \mathrm{p} .13]$ of the lattices $C\left(X_{1}\right)$ and $C\left(X_{2}\right)$. We shall now prove the less trivial converse.

THEOREM 2. Let $R$ be a chain with neither a maximal nor minimal element, and let it be endowed with its order topology. Let $X$ be a compact space and $C=C(X)$ the lattice of all continuous functions from $X$ to $R$. 
Suppose $C$ is a direct product of lattices, $C \cong C_{1} \times C_{2}$. Then $X$ splits into two open and closed sets $X_{1}, X_{2}$ in such a fashion that $C_{i} \cong C\left(X_{i}\right)$.

Proof. We first remark that any prime ideal $P$ in $C$ is of the form $P_{1} \times C_{2}$ or $C_{1} \times P_{2}$, where $P_{i}$ is a prime ideal in $C_{i}$. This follows readily from Theorem 10 of [8], or it can be proved by repeating the argument of Lemma 3, if we replace $X$ by a two-point space and $R$ by $C_{1}$ at one point and $C_{2}$ at the other. Let us write $Z_{1}$ for the class of prime ideals of the form $P_{1} \times C_{2}, Z_{2}$ for those of the form $C_{1} \times P_{2}$.

Now take any point $x$ in $X$ and any prime ideal $P$ associated with it. We define $X_{i}$ to be the set of those $x$ for which $P \in Z_{i}$. It follows from Lemma 4 that this definition makes sense, since any two prime ideals associated with the same point must fall into the same class.

Next we show that $X_{1}$ is closed. If not, there is a point $y \in X_{2}$ which is in the closure of $X_{1}$. By the first half of Lemma 7, $A\left(X_{1}\right)$ is contained in a prime ideal $P$ associated with $y$. But $P$ is of the form $C_{1} \times P_{2}$ while $A\left(X_{1}\right)$ is of the form $B \times C_{2}$, so this is impossible. Similarly, $X_{2}$ is closed, and each is thus open and closed.

We shall now set up a lattice isomorphism between $C_{1}$ and $C\left(X_{1}\right)$. Given $c_{1} \in C_{1}$, we pair it with any $c_{2}$ in $C_{2}$, obtaining a function $f \in C$, whose specialization to $X_{1}$ we shall call $f_{1}$. We prove that the correspondence is order-preserving, which incidentally shows it to be well defined. Suppose we have $d_{1} \in C_{1}$ with $d_{1} \leqq c_{1}$ and that $d_{1}$ has given rise to the function $g$ on $X$ specializing to $g_{1}$ on $X_{1}$. If $g_{1}(x)>f_{1}(x)$ at some point $x \in X_{1}$, we can build a prime ideal $P$ associated with $x$ such that $f \in P, g \in C-P$. But $P$ is of the form $P_{1} \times C_{2}$ and hence $f \in P$ implies $g \in P$, since the $C_{1}$-component of $f$ exceeds that of $g$. This contradiction shows that $f_{1} \geqq g_{1}$, as desired.

To set up the correspondence the other way we take a function $f_{1}$ on $X_{1}$, pair it with any $f_{2}$ on $X_{2}$, to obtain a function $f$ whose $C_{1}$-component is, say, $c_{1}$. Suppose $g_{1} \leqq f_{1}$ and that $g_{1}$ gives rise to $d_{1} \in C_{1}$. We assert that $d_{1} \leqq c_{1}$. For if not, we note that $C_{1}$ is a distributive lattice, whence [4, Theorem 5.8] there exists a prime ideal $P_{1} \subset C_{1}$ with $c_{1} \in P_{1}, d_{1} \in C_{1}-P_{1}$. For the corresponding prime ideal $P=P_{1} \times C_{2}$ we have $f \in P, g \in C-P$. But $P$ is associated with a point in $X_{1}$; by Lemma 6 we have a contradiction.

There remains the trivial observation that the two correspondences we have defined are inverse to each other, and we have completed the proof of Theorem 2.

Remark. It is to be observed that no separation axiom is required for Theorem 2. In case $R$ is the reals, we can even drop the assumption of compactness by making use of the Stone-Čech compactification 
$\beta(X)$. (Cf. [5, p. 578].) But for the general case no such device is available, and the validity of Theorem 2 for non-compact spaces remains undecided.

5. Rings and Banach spaces. Suppose we are given $C(X)$ as the ring of real continuous functions. Now $f \geqq g$ is equivalent to the statement that $f-g$ is a square. Hence we are also given the lattice; the lattice characterization subsumes the ring characterization.

Suppose we are given $C(X)$ as a Banach space, with $X$ a compact Hausdorff space. Let $e$ be an extreme point on the unit sphere, that is, a point which is not an interior point of a segment lying in the unit sphere. It is easy to see that $e$ can assume only the values 1 and -1 ; suppose it is 1 on $Y_{1}$ and -1 on $Y_{2}$, where $Y_{1}, Y_{2}$ are open and closed disjoint sets whose sum is $X$. Now a function $f \neq 0$ which satisfies $\|f /\| f\|-e\| \leqq 1$ must be non-negative on $Y_{1}$ and non-positive on $Y_{2}$. Hence if we write $f \geqq 0$ whenever it fulfills this condition, we preserve order on $Y_{1}$ and invert order on $Y_{2}$, as compared with the natural order in $C(X)$. This gives us a lattice which is at any rate isomorphic to $C(X)$. Hence $C(X)$ as a Banach space determines $C(X)$ as a lattice, and the lattice characterization subsumes the Banach space characterization.

Finally we remark that in our Theorem 2 we have likewise subsumed the analogous ring and Banach space theorems, in the following strong sense: if we have $C(X)$ expressed as a direct sum of rings or Banach spaces, then that very decomposition expresses the lattice as a direct product (it being understood in the Banach space case that the lattice being decomposed is the one obtained after inversion of order on $Y_{2}$ ). We omit the straightforward proof of this fact.

6. Chains with 0 and $I$. If $R$ is a chain which has a minimal or maximal element or both, Theorems 1 and 2 remain valid, with the proviso that $R$-separation in the hypothesis of Theorem 1 is replaced by $R$-normality. In several of the proofs, however, there occur technical complications. We have preferred to avoid these complications, which add nothing to the fundamental idea. We shall merely mention the two main modifications that are necessary: (1) The definition of "equivalence" of prime ideals is revised to read: either their intersection contains a prime ideal, or the intersection of their complements contains a dual prime ideal. (2) In the characterization of the topology, $x$ is in the closure of $S$ if and only if $A(S)$ is contained in a prime ideal, or the object dual to $A(S)$ is contained in a dual prime ideal. 


\section{BIBLIOGRAPHY}

1. R. F. Arens, A topology for spaces of transformations, Ann. of Math. vol. 47 (1946) pp. 480-495.

2. R. F. Arens ond J. L. Kelley, Characterizations of the space of continuous functions over a compact Hausdorff space, To be published in Trans. Amer. Math. Soc.

3. S. Banach, Theorie des operations lineaires, Warsaw, 1932.

4. G. Birkhoff, Lattice theory, Amer. Math. Soc. Colloquium Publications, vol. 25, New York, 1940.

5. S. Eilenberg, Banach space methods in topology, Ann. of Math. vol. 43 (1942) pp. 568-579.

6. I. Gelfand and A. N. Kolmogoroff, On rings of continuous functions on a topological space, C. R. (Doklady) URSS. vol. 22 (1939) pp. 11-15.

7. M. H. Stone, Applications of the theory of Boolean rings to general topology, Trans. Amer. Math. Soc. vol. 41 (1937) pp. 375-481.

8. - Topological representations of distributive lattices and Brouwerian logics, Casopis Matematiky a Fysiky vol. 67 (1937) pp. 1-25.

9. - A general theory of spectra II, Proc. Nat. Acad. Sci. U.S.A. vol. 27 (1941) pp. 83-87.

University of Chicago

\section{LINEAR TOPOLOGICAL DIVISION ALGEBRAS}

RICHARD ARENS

1. Introduction. We present a generalization of the familiar theorem of Frobenius that any finite-dimensional linear division algebra $A$ over the real number field is isomorphic to the real, the complex, or the quaternion number system. The generalization consists in replacing the hypothesis of finite dimensionality of $A$ by the weaker hypothesis that $A$ be a complete linear space with a topology in which multiplication is continuous and which is based on a countable system of convex open sets.

Previous generalizations of Frobenius' theorem have been indicated or proved by S. Mazur [6], ${ }^{1}$ G. Silov [8], and I. Gelfand [4]. These writers have generally assumed that $A$ have a norm; and only that case has been adequately considered in which scalar multiplication by complex numbers is assumed. We shall give the proof of the general case without limiting ourselves to the commutative case or to complex scalars. 1946.

Presented to the Society August 23, 1946; received by the editors November 20,

${ }^{1}$ Numbers in brackets refer to the bibliography at the end of the paper. 